\title{
Ultra Wideband Radar Target Discrimination Using the Signatures Algorithm
}

\author{
Andrey Baev, Yury Kuznetsov, Andrey Aleksandrov \\ Moscow Aviation Institute (State University of Aerospace Technology) \\ Theoretical Radioelectronics Department \\ Volokolamskoe Shosse 4, GSP, 125993, Moscow, Russia \\ E-mail: maik405@mtu-net.ru \\ Tel.: +0070951584047
}

\begin{abstract}
An aspect independent radar target discrimination method based on the natural frequencies of ultra wideband radar targets is introduced. The sets of points in multi-dimension space corresponding to the poles on a complex plane (natural resonances) were offered as signatures of radar targets. This approach allows performing an automated discrimination algorithm of radar targets. The results of experimental research of the signals scattered by the scaled aircraft models by using the signatures algorithm are presented.
\end{abstract}

\section{INTRODUCTION}

In recent years, many radar target discrimination methods using the ultra wideband response of a target to a transient incident waveform have stimulated growing interest [1], [2]. The Baum's singularity expansion method (SEM) [3] provides the necessary mathematical formulation for describing the transient behavior of conducting targets. The late-time response of the target can be represented as a sum of natural modes,

$$
\begin{aligned}
& y[n]=x[n]+w[n]= \\
& =\sum_{k=1}^{K} A_{k} \mathrm{e}^{-\sigma_{k} n T_{\mathrm{o}}} \cos \left(2 \pi f_{k} n T_{\mathrm{o}}+\varphi_{k}\right)+w[n],
\end{aligned}
$$

where $s_{k}=\sigma_{k}+j 2 \pi f_{k}$ is the $k$-th aspect-independent natural complex frequency of the target, and $A_{k}$ and $\varphi_{k}$ are the aspect- and excitation-dependent amplitude and phase of the $k$-th target mode, respectively, $w[n]$ are samples of additive Gaussian band-limited noise. $T_{o}$ describes the period of sampling. The number of natural resonanses $K$ is determined by the finite frequency content of the waveform exciting the target and by the geometrical shape of the object.

Traditional parametrical methods can be used for ultra wideband radar target parameter estimation of finite quantity of parameters describing an object, for example, the well-known Prony's method, pencil-of-function method, ESPRIT, etc [4].

The discrimination technique using signatures algorithm is described in this paper. Discrimination of ultra wideband radar targets is performed by using objects' signatures. These signatures are the points in multidimensional attribute space, each axis of which is proportional to the true value of poles' coordinates on a complex $z$-plane for the expected target. The distance between an estimation of a point in attribute space of the identified object and the signatures of objects stored in a databank is the criterion for its discrimination. Such approach allows creating the automated system of ultra wideband radar target discrimination.

The paper is organized as follows. In section II, the resonant model of ultra wideband radar targets is presented. Section III describes the radar target discrimination algorithm. The results of experimental research of signals scattered by the scaled aircraft models are presented in Section IV. Concluding remarks are drawn in Section V.

\section{THE RESONANT MODEL}

One of the major problems during to synthesis of discrimination algorithms is the representation of the initial data by some model. The choice of a concrete method of model's formation for objects and signals should provide the compromise between accuracy of reproduction of scattered fields and simplicity of computing.

In accordance with Baum's singularity expansion method the electromagnetic radar target response can be decomposed into a finite sum of damped sinusoids. So the natural electromagnetic scattering of objects can be described by the resonant model. This model consists of parameters of two types: dependent on an exciting signal (polarization, form, direction of arrival) and the natural complex resonant frequencies of targets. These frequencies are determined by basic geometrical sizes and shape of objects, practically do not depend on an aspect angle and can be used for discrimination of the radar targets.

According to the SEM, system transfer function of object completely characterized by a set of natural resonant frequencies on a complex $s$-plane. The resonant frequencies band of radar targets with the characteristic sizes from 0,1 up to 10 meters are in the range from units up to hundreds of megahertz.

To make the analysis of ultra wideband radar target discrimination algorithm we have selected resonance frequencies [2], appropriate to resonances of scaled models of aircrafts F-4 and MIG-27. Fig. 1 shows the locations of resonant poles on the complex $z$-plane for these targets. The relative values of poles

$$
z_{k}=\exp \left\{\left(\sigma_{k}+j 2 \pi f_{k}\right) T_{o}\right\}
$$




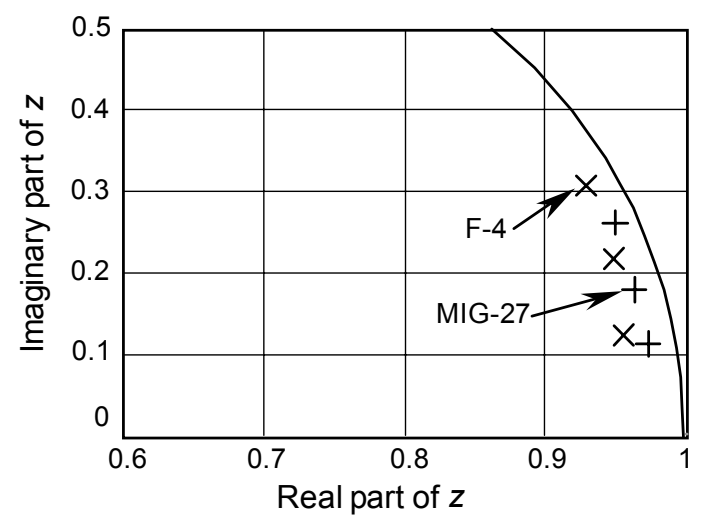

Fig. 1. Complex z-plane plot of aircraft models

Fig. 2 shows the power spectrum of these resonant models. The distinction in a power spectrum of these models allows making the conjecture of possibility to discriminate radar targets by using natural frequencies of these objects.

\section{RADAR TARGET DISCRIMINATION ALGORITHM}

In this paper the following task is considered. It is necessary to discriminate the given number of radar targets by using measured ultra wideband responses of objects. It is supposed, that these objects are a priori divided into $M$ classes. The set of the measured natural poles $z_{1}, z_{2}, \ldots, z_{N}$ is used as attributes of the chosen targets' classes, or as a dictionary of attributes.

The quantity of attributes $N$ (the dimension of the attributes' dictionary) is determined by the given set of classes and depends on used discrimination algorithm. The expression determining classes of radar targets can be written in language of these attributes.

Generally it is necessary to minimize the attributes quantity $N$, which is describing the object, because it simplifies discrimination algorithm of ultra wideband radar targets. Definition of optimum number $N$ is one of tasks of our research.

A point in $\mathrm{N}$-dimensional space of attributes represents each class of identified objects

$$
\bar{S}_{j}=\left\{S_{j 1}, S_{j 2}, \ldots, S_{j N}\right\},
$$

where $S_{j i}$ is the appropriate coordinate in space of attributes, $j=1 \ldots M$.

The measured set of attributes for the real response from ultra wideband radar target differs from each set of classes' attributes, as the signal is corrupted by noise:

$$
\bar{Y}_{j}=\bar{S}_{j}+\bar{W}
$$

where $\bar{Y}_{j}$ is the set of measured attributes $\bar{Y}_{j}=\left\{Y_{j 1}, Y_{j 2}\right.$, $\left.\ldots, Y_{j N}\right\}, \bar{W}$ is an error in attributes' estimation because the noise is present in the received signal.

The correspondence of the measured set of attributes $\bar{Y}_{j}$ to one of the given classes of the targets $\bar{S}_{j}$ (or the decision criterion) is following. The space of attributes is divided into $M$ of the not crossed areas appropriate to chosen classes of the targets. The borders of these areas are determined by the solution of optimization problem:

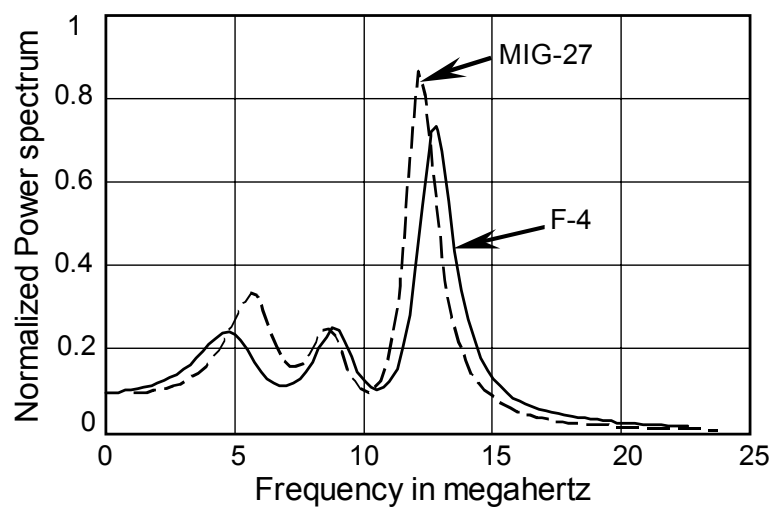

Fig. 2. Power spectrum of resonant models

decision $\Gamma_{j}$ on identification of $j$-th class of the targets is accepted for set of the measured parameters $\bar{Y}_{i}$ in case if the distances $R_{i}$ between $\bar{Y}_{i}$ and $\bar{S}_{j}$ in space of attributes is minimally in comparison with distances up to all other signatures:

$$
R_{i}=\left|\bar{Y}_{i}-\bar{S}_{j}\right|, \quad j=1, \ldots, M
$$

Quality of ultra wideband radar targets discrimination algorithm is estimated by probability of true discrimination for the targets of all classes:

$$
P_{\text {true }}=\sum_{i=1}^{M} P_{i} \cdot P\left(\Gamma_{i} / H_{i}\right),
$$

where $P_{i}$ is a priori probability of $i$-th class, $P\left(\Gamma_{i} / H_{i}\right)$ is the conditional probability of decision making $\Gamma_{i}$ provided that is put forward hypothesis $H_{i}$ about identification of the $i$-th class is true.

The probability of true discrimination is determined by dimension of the dictionary of attributes, i.e. the quantity of the measured natural poles of the target, and depends on noise level $\mathrm{w}[\mathrm{n}]$ present in the data. Therefore it is important to define of the boundary signal-to-noise ratio at which the given level of probability of true discrimination is provided.

The probability of true discrimination at the given signal-to-noise ratio depends on the number of significant attributes $N$ and on their concrete choice from $K$ measured natural frequencies of discriminations target. The influence of the number of significant attributes and of the concrete parameters of resonant model for chosen value $N$ on the probability of true discrimination is very interesting.

\section{RESUlts of Digital Modelling}

For an experimental research of ultra wideband radar target discrimination algorithm the responses of the scaled models of planes F-4 and MIG-27, described in section II, were used. Independent Gaussian band-limited noise sequence each run was perturbed. The signal-tonoise ratio SNR was estimated as

$$
\mathrm{SNR}=10 \cdot \lg \left(\frac{\mathrm{M}\left(x_{k}^{2}\right)}{\mathrm{M}\left(n_{k}^{2}\right)}\right),
$$

where $\mathrm{M}(\bullet)$ denotes the mean value. 


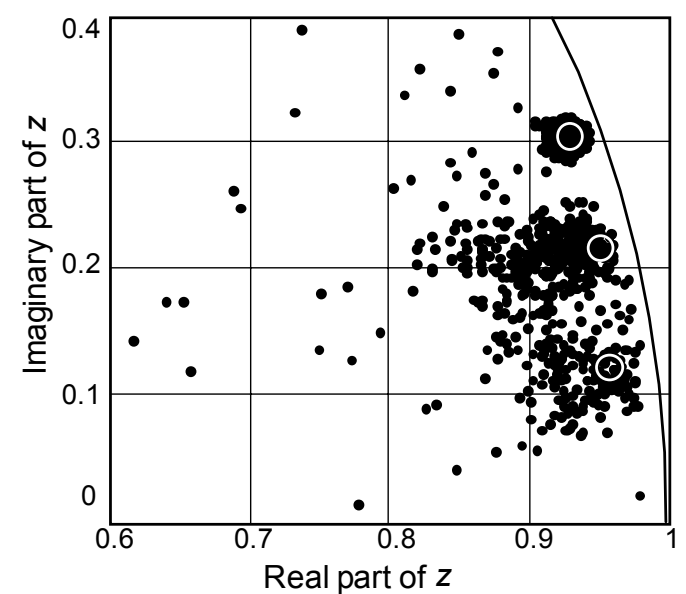

Fig. 3. Estimates of poles for 500 independent runs using pencil-of-function method, $\mathrm{SNR}=10 \mathrm{~dB}$

The results of F-4 resonant model poles estimation by pencil-of-function method for 500 independent realizations of signal with Gaussian band-limited noise with signal-to-noise ratio $\mathrm{SNR}=10 \mathrm{~dB}$ are depicted in Fig. 3 . The true signal poles locations are depicted as circles, the estimations are shown as points. From figure it is visible, that estimations for high-frequency (HF) poles are concentrated near the true value while estimations of midfrequency (MF) and low-frequency (LF) poles have much more dispersion.

The set of the measured natural poles on complex plane were used as information parameters in a dictionary of attributes for the radar targets. The resonant models of both targets consist of three pairs of complex conjugate poles, so a maximum quantity of attributes is $N=6$.

At the first stage of digital modeling we analyses dependence of probability of true discrimination of radar targets on the signal-to-noise ratio at different quantity of the information attributes $N$, depicted on fig. 4. From figure it is visible, that at reduction $N$ for the fixed signalto-noise ratio the probability of true discrimination is increased. It shows that the task of discrimination of ultra wideband radar targets may be solved by the best way at $N=2$, if the HF pole is present in each case.

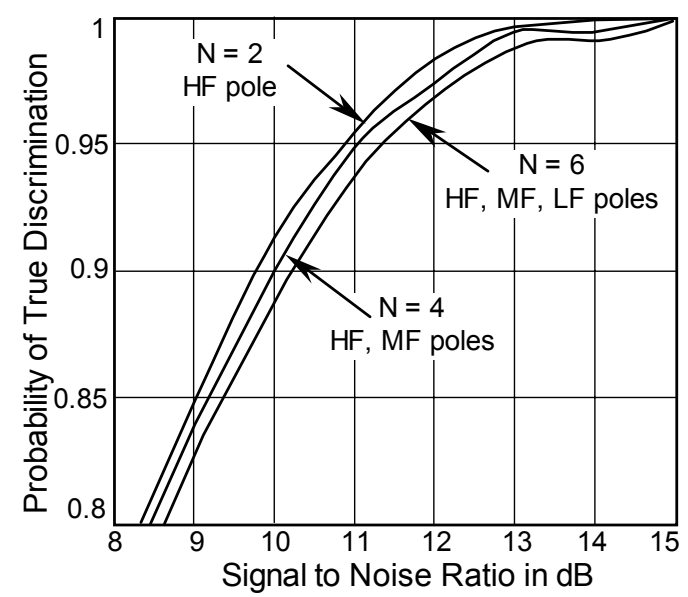

Fig. 4. Probability of true discrimination for several numbers of quantities of attributes $N$

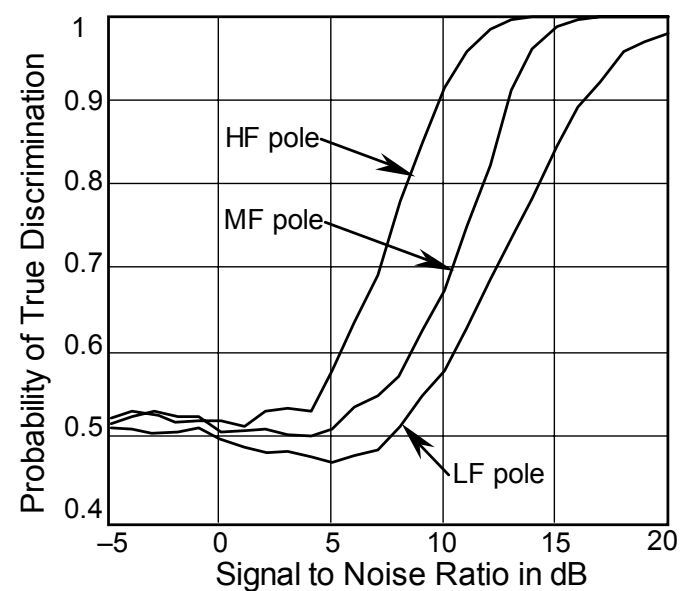

Fig. 5. Probability of true discrimination for single poles of resonant models

Further dependence of probability of true discrimination of two radar targets was researched at $N=2$ for LF, $\mathrm{MF}$ and HF poles of resonant model. Discrimination was carried out for each type of a pole independently. Probabilities of true discrimination of the radar targets from the signal-to-noise ratio for the specified types of poles are presented on Fig. 5. From figure it is visible, that the maximal probability of true discrimination is provided with a high-frequency pole. It is possible to explain this by that the high frequency pole in our model is corresponds to the higher level of Q-factor. The Q-factor of a resonant model's pole is the following ratio:

$$
\mathrm{Q}_{k}=\frac{\omega_{k}}{2\left|\sigma_{k}\right|}=\frac{\pi f_{k}}{\left|\sigma_{k}\right|}
$$

Dependence of probability of true discrimination of radar targets on Q-factor of poles for the fixed values signal-to-noise ratio was analyzed and depicted on Fig. 6. From figure it is visible, that for the higher values of signal-to-noise ratio the true discrimination of radar targets is possible with Q-factor of poles about 2-3. At small values of signal-to-noise ratio the acceptable level of probability of true discrimination is achieved at Q-factor of poles more than 6 .

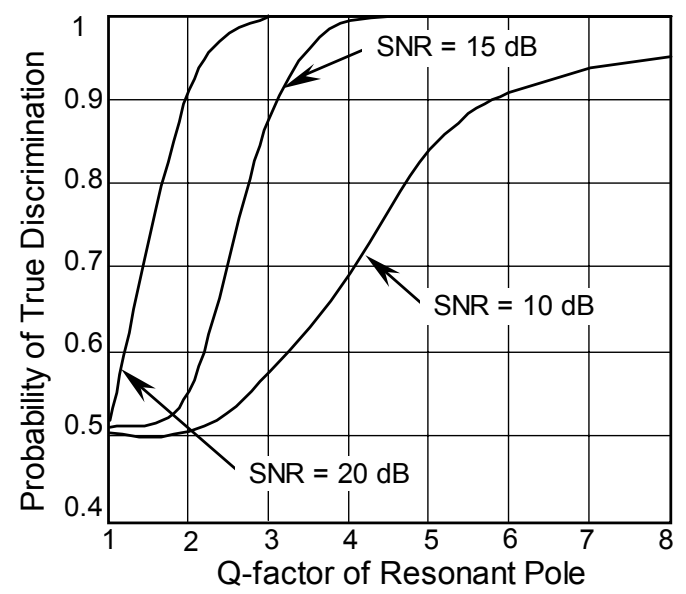

Fig. 6. Probability of true discrimination for one pole of resonant model 


\section{CONCLUSIONS}

An aspect independent radar target discrimination method based on the natural frequencies of ultra wideband radar targets is introduced. The sets of natural resonances were offered to use as signatures of radar targets. These signatures are the points in many-dimensional attribute space, each axis of which is proportional to the true value of poles' coordinate on a complex $z$-plane for the expected target.

The distance between an estimation of a point in attribute space for the identified object and the signatures of objects stored in a databank is the criterion for its discrimination. Such approach allows creating the automated system of ultra wideband radar target discrimination.

The main results of our researches are follow:

- for the chosen resonant models of scaled planes F-4 and the MIG-27 it is shown, that the increase of dimension of attributes' space results in deterioration of probability of true discrimination. So, for the signal-to-noise ratio $\mathrm{SNR}=10 \mathrm{~dB}$ reduction $N$ from 6 up to 2 leads to increasing $P_{\text {true }}$ from 0.88 up to 0.92 ;

- at the given quantity of information attributes $N=2$ probability of true discrimination depends on the chosen pole. So, for the signal-to-noise ratio $\mathrm{SNR}=10 \mathrm{~dB}$ the gain due to a correct choice of a pole may reach 0.33 , i.e. from $P_{\text {true }}=0.57$ up to 0.9 ;

- the dependence of probability of true discrimination on Q-factor of poles of resonant model and SNR is researched. For example, if the probability of true discrimination is 0.9 and the Q-factor will change from 2 up to 6 then required SNR will decrease from 20 down to $10 \mathrm{~dB}$.

\section{REFERENCES}

[1] Taylor, James D., Introduction to Ultra-Wideband Radar Systems / editor Taylor, James D., CRC Press, 1995.

[2] Yury Kuznetsov, Andrey Baev, Rudolf Sedletskiy, "Application of E-pulse Method for Remote Sensing Arbitrary Shaped Objects in Lossy Media," in $30^{\text {th }}$ European Microwave Conference, pp. 255-259, Oct. 2000.

[3] C.E. Baum, "The Singularity Expansion Method," in Transient Electromagnetic Fields, L.B. Felson, Ed. New York: Springer-Verlag, 1976.

[4] Yury Kuznetsov, Andrey Baev, Vitali Chtchekatourov, "Parameter Estimation of the Resonant Model in Passive and Active Radar Systems by Using Third-Order Statistics," in $29^{\text {th }}$ European Microwave Conference, pp. 395398, Oct. 1999. 